\title{
Ultrathin-Body High-Mobility InAsSb-on-Insulator Field-Effect Transistors
}

\author{
Hui Fang, Steven Chuang, Kuniharu Takei, Ha Sul Kim, Elena Plis, Chin-Hung Liu, \\ Sanjay Krishna, Yu-Lun Chueh, and Ali Javey
}

\begin{abstract}
Ultrathin-body InAsSb-on-insulator n-type fieldeffect transistors (FETs) with ultrahigh electron mobilities are reported. The devices are obtained by the layer transfer of ultrathin $\mathrm{InAs}_{0.7} \mathrm{Sb}_{0.3}$ layers (thickness of 7-17 nm) onto $\mathrm{Si} / \mathrm{SiO}_{2}$ substrates. InAsSb-on-insulator FETs exhibit an effective mobility of $\sim 3400 \mathrm{~cm}^{2} / \mathrm{V} \cdot \mathrm{s}$ for a body thickness of $7 \mathrm{~nm}$, which represents $\sim 2 \times$ enhancement over InAs devices of similar thickness. The top-gated FETs deliver an intrinsic transconductance of $\sim 0.56 \mathrm{mS} / \mu \mathrm{m}$ (gate length of $\sim 500 \mathrm{~nm}$ ) at $V_{\mathrm{DS}}=0.5 \mathrm{~V}$ with $I_{\mathrm{ON}} / I_{\mathrm{OFF}}$ of $10^{2}-10^{3}$. These results demonstrate the utility of the transfer process for obtaining high-mobility n-FETs on Si substrates by using mixed anion arsenide-antimonide as the active channel material.
\end{abstract}

Index Terms-Field-effect transistors (FETs), InAsSb, ultrathin body (UTB), XOI.

\section{INTRODUCTION}

$\mathbf{H}$ IGH-MOBILITY semiconductors show great promise as the channel material of ultrafast low-power field-effect transistors (FETs) and have been actively explored in the past few decades [1]-[4]. Among all known semiconductors, mixed anion $\operatorname{InAs}_{x} \mathrm{Sb}_{1-x}$ has one of the highest electron mobilities and saturation velocities [5]. However, it also has one of the smallest bandgaps [5], [6]. For such devices, ultrathin-body (UTB) architectures are essential to enable acceptable leakage currents. Conventionally, $\operatorname{InAs}_{x} \mathrm{Sb}_{1-x}$ devices have been

Manuscript received December 19, 2011; accepted January 18, 2012. Date of publication March 2, 2012; date of current version March 23, 2012. This work was supported in part by Focus Center Research Program/Materials Structures, and Devices, by the National Science Foundation (NSF) E3S Center, by NSF Center Of Integrated Nanomechanical Systems, and by the Air Force Office of Scientific Research under Award FA9550-10-1-0113. The material characterization part of this work was supported in part by the Director, Office of Science, Office of Basic Energy Sciences, Division of Materials Sciences and Engineering of the U.S. Department of Energy under Contract De-Ac0205Ch11231 and in part by the Electronic Materials program. The work of Y.-L. Chueh was supported by the National Science Council, Taiwan, through Grant NSC 98-2112-M-007-025-MY3. The work of A. Javey was supported in part by a Sloan Research Fellowship, by an NSF CAREER Award, and by the World Class University program at Sunchon National University. The review of this letter was arranged by Editor M. Passlack.

H. Fang, S. Chuang, K. Takei, H. S. Kim, and A. Javey are with the Department of Electrical Engineering and Computer Sciences, University of California, Berkeley, CA 94720 USA (e-mail: huifang@berkeley.edu; s_chuang@eecs.berkeley.edu; ktakei@berkeley.edu; hasul@berkeley.edu; ajavey@eecs.berkeley.edu).

E. Plis and S. Krishna are with the Department of Electrical and Computer Engineering, The University of New Mexico, Albuquerque, NM 87106 USA (e-mail: alien@chtm.unm.edu; skrishna@chtm.unm.edu).

C.-H. Liu and Y.-L. Chueh are with the Department of Materials Science and Engineering, National Tsing Hua University, Hsinchu 30013, Taiwan (e-mail: isaacyahoo@gmail.com; ylchueh@mx.nthu.edu.tw).

Color versions of one or more of the figures in this letter are available online at http://ieeexplore.iee.org.

Digital Object Identifier 10.1109/LED.2012.2185477 fabricated as complex quantum well structures on III-V or $\mathrm{Si}$ substrates. While the devices exhibited promising initial results, due to leakage from gate and/or not fully depleted body, they suffered from high $I_{\mathrm{OFF}}$ [7]-[10]. In consideration of supporting substrates, $\mathrm{Si}$ is a well-established material and is highly preferred over III-V semiconductors. However, using direct MBE growth to integrate both $\mathrm{n}$ - and $\mathrm{p}$-channel materials onto $\mathrm{Si}$ for CMOS will be very challenging due to the large lattice mismatch between different desired materials and $\mathrm{Si} / \mathrm{SiO}_{2}$. Previously, we demonstrated the transfer of InAs ultrathin membranes onto $\mathrm{Si} / \mathrm{SiO}_{2}$ substrates to form high-performance n-type FETs (n-FETs), termed "XOI" in analogy to the wellestablished silicon-on-insulator field. The mobility of InAs XOI devices was found to be as high as $\sim 5000 \mathrm{~cm}^{2} / \mathrm{V} \cdot \mathrm{s}$ for body thicknesses of $\sim>20 \mathrm{~nm}$ and decreases to $\sim 1600 \mathrm{~cm}^{2} / \mathrm{V} \cdot \mathrm{s}$ when scaled down to $8 \mathrm{~nm}$ in thickness [11]. Here, we extend the XOI concept to $\operatorname{InAs}_{x} \mathrm{Sb}_{1-x}$ as a demonstration of even higher mobility III-V FETs, particularly for UTB thicknesses of $<10 \mathrm{~nm}$ which are required for scaled transistors based on small-bandgap semiconductors.

\section{EXPERIMENTS}

First, ultrathin $\mathrm{InAs}_{0.7} \mathrm{Sb}_{0.3}$ layers of different thicknesses $\left(T_{\text {InAsSb }}=7\right.$ and $\left.17 \mathrm{~nm}\right)$ were transferred onto $\mathrm{Si} / \mathrm{SiO}_{2}$ substrates following the epitaxial layer transfer technique described previously [11]. InAs $\mathrm{Is}_{0.7} \mathrm{Sb}_{0.3}$ was grown on a $60-\mathrm{nm}$ $\mathrm{Al}_{0.4} \mathrm{Ga}_{0.6} \mathrm{Sb}$ sacrificial layer on a GaSb substrate by molecular beam epitaxy. The InAsSb layer was then pattern etched by using a mixture of citric acid $(1 \mathrm{~g} / \mathrm{mL}$ of water) and hydrogen peroxide (30\%) at 1:20 volume ratio (etch rate of $\sim 0.7 \mathrm{~nm} / \mathrm{s}$ ), and the AlGaSb layer was selectively etched by ammonium hydroxide (1.5\% in water). $\mathrm{Ni}\left(T_{\mathrm{Ni}}=40 \mathrm{~nm}\right)$ source $(\mathrm{S})$ and drain (D) electrodes were fabricated using lithography and metallization. For top-gated FETs, a 10 -nm-thick $\mathrm{ZrO}_{2}$ gate dielectric was deposited by atomic layer deposition at $115^{\circ} \mathrm{C}$, followed by a forming gas anneal at $150{ }^{\circ} \mathrm{C}$ for $10 \mathrm{~min}$. Subsequently, Ni top-gate (G) electrodes, overlapping the S/D, were fabricated. Fig. 1(a) shows a TEM image of the 7-nm InAsSb layer on $\mathrm{Si} / \mathrm{SiO}_{2}$ with a $\mathrm{Ni} / \mathrm{ZrO}_{2}$ high- $\kappa$ stack, while the HRTEM image in Fig. 1(b) shows the single crystallinity of the InAsSb channel, exhibiting highly abrupt interfaces between InAsSb and $\mathrm{Si} / \mathrm{SiO}_{2}$ without any visible voids.

\section{RESUlts And Discussions}

In order to probe the electrical properties of InAsSb XOI FETs, back- and top-gated devices with varied gate lengths $\left(L_{G}\right)$ were fabricated and characterized. For back-gated FETs, 50-nm-thick thermally grown $\mathrm{SiO}_{2}$ was used as the gate 


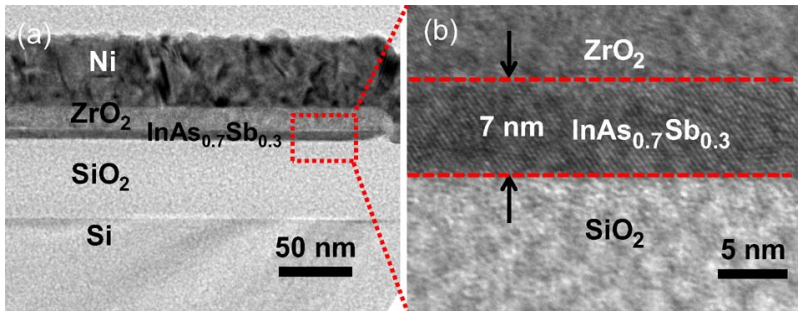

Fig. 1. (a) TEM image of an $\sim 7$-nm-thick $\operatorname{InAs}_{0.7} \mathrm{Sb}_{0.3}$ XOI (body oxide thickness of $50 \mathrm{~nm}$ ) substrate. The nanoribbon is coated with a $\mathrm{ZrO}_{2} / \mathrm{Ni}$ bilayer ( $\sim 25$ and $\sim 50 \mathrm{~nm}$, respectively). (b) HRTEM image showing the single-crystal structure of an InAs $0.7 \mathrm{Sb}_{0.3}$ nanoribbon with atomically abrupt interfaces with $\mathrm{ZrO}_{2}$ and $\mathrm{SiO}_{2}$ layers on the top and bottom surfaces, respectively.
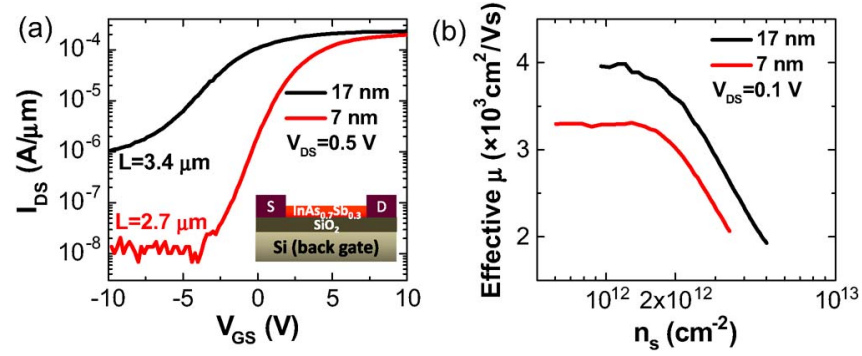

Fig. 2. (a) Typical back-gate $I_{\mathrm{DS}}-V_{\mathrm{GS}}$ for 7 - and 17 -nm-thick $\operatorname{InAs}_{0.7} \mathrm{Sb}_{0.3}$ XOI n-FETs on $50-\mathrm{nm} \mathrm{SiO}_{2}$ at $V_{\mathrm{DS}}=0.5 \mathrm{~V}$. The inset shows the schematic of the back-gated devices. (b) Effective mobility extracted from $I_{\mathrm{DS}}-V_{\mathrm{GS}}$ characteristics at $V_{\mathrm{DS}}=0.1 \mathrm{~V}$

dielectric. Fig. 2(a) shows the transfer characteristics of the 7- and 17-nm-thick InAsSb XOI FETs at $V_{\mathrm{DS}}=0.5 \mathrm{~V}$, for $L_{G}=2.7$ and $3.4 \mu \mathrm{m}$, respectively. The back-gated 7-nm-thick device exhibits an $I_{\mathrm{ON}} / I_{\mathrm{OFF}}$ ratio of $\sim 10^{4}$, which is more than two orders of magnitude greater than that of the 17-nm device. This significant improvement in OFF current can be attributed to better electrostatic control from gating a thinner body [12]. The raising of bandgap by confinement will also contribute to a lower OFF current, since the barrier for thermionic emission of carriers would be higher. Specifically, since InAsSb has a large Bohr radius (between 34 and $65 \mathrm{~nm}$, which are for bulk InAs and InSb, respectively [13]), heavy quantum confinement is expected in UTB membranes. An approximate expression for the ground-state energy of electrons and holes can be derived by solving the 1-D Schrodinger equation for a finite potential well. The effective bandgaps would be 0.6 and $0.32 \mathrm{eV}$ for 7- and 17-nm InAs $\mathrm{I}_{0.7} \mathrm{Sb}_{0.3}$, respectively. Fig. 2(b) shows the extracted effective mobilities $\left(\mu_{\mathrm{eff}}\right)$ as a function of the 2-D carrier density at $V_{\mathrm{DS}}=0.1 \mathrm{~V}$ for the long-channel back-gated FETs (with $T_{\mathrm{ox}}=50 \mathrm{~nm}$ ) shown in Fig. 2(a). The mobility was obtained from the expression

$$
\mu_{\mathrm{eff}}=\frac{\partial I_{\mathrm{DS}}}{\partial V_{\mathrm{DS}}} \frac{L_{G}}{C_{\mathrm{ox}}\left(V_{G}-V_{T}-0.5 V_{\mathrm{DS}}\right)}
$$

where $V_{T}$ is the threshold voltage extracted from the linear $I_{\mathrm{DS}}-V_{\mathrm{GS}}$ curve and $C_{\mathrm{ox}}=\varepsilon_{\mathrm{ox}} \varepsilon_{0} / T_{\mathrm{ox}}$ is the gate oxide capacitance per unit area $\left(\varepsilon_{\mathrm{ox}} \sim 3.9\right.$ is the dielectric constant of $\mathrm{SiO}_{2}$, $\varepsilon_{0}$ is the vacuum permittivity, and $T_{\mathrm{ox}}=50 \mathrm{~nm}$ is the $\mathrm{SiO}_{2}$ thickness). Here, we utilized the simple parallel-plate model, and the effects of quantum capacitance and fringe field are ignored, which is a valid assumption given the thick gate oxide (i.e., small oxide capacitance) of the back-gated devices and that the channel width is much greater than thickness.
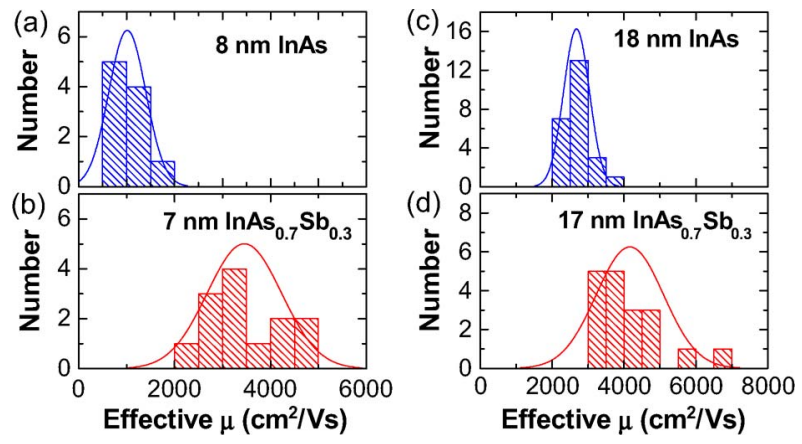

Fig. 3. Histogram plots of effective mobility (at $n_{s}=2 \times 10^{12} \mathrm{~cm}^{-2}$ ) in InAs and InAsSb XOI n-FETs of (a) $T_{\text {InAs }}=8 \mathrm{~nm}$, (b) $T_{\text {InAs } 0.7 \mathrm{Sb} 0.3}=$ $7 \mathrm{~nm}$, (c) $T_{\text {InAs }}=18 \mathrm{~nm}$, and (d) $T_{\text {InAs } 0.7 \mathrm{Sb} 0.3}=17 \mathrm{~nm}$.

The effective mobility histograms extracted for long-channel $\mathrm{InAs}_{0.7} \mathrm{Sb}_{0.3}$ (thicknesses of 7 and $17 \mathrm{~nm}$ ) and InAs (thicknesses of 8 and $18 \mathrm{~nm}$ ) XOI FETs are shown in Fig. 3. Note that the dimensions (including channel width $W$, which is typically $\sim 320-380 \mathrm{~nm}$ ) of each device were directly measured by SEM and used to normalize the current and extract the mobilities. InAsSb devices exhibit average effective mobilities of $\sim 3400$ and $\sim 4100 \mathrm{~cm}^{2} / \mathrm{V} \cdot \mathrm{s}$ at $n_{s}=2 \times 10^{12} \mathrm{~cm}^{-2}$ for the 7- and 17-nm thicknesses, respectively. Note that the mobility degradation with thickness is mainly due to enhancement of surface roughness and surface polar phonon scattering [14]. These mobility values present $\sim 2 \times$ enhancement over InAs XOI FETs with similar thicknesses (Fig. 3). The variation of the mobility may be caused by the different amount of interface trap states $\left(D_{\text {it }}\right)$ introduced during processing. This mobility improvement coincides with the previously reported Hall mobility difference between $\operatorname{InAs}_{0.7} \mathrm{Sb}_{0.3}\left(\mu_{\text {Hall }} \sim 42000 \mathrm{~cm}^{2} / \mathrm{V} \cdot \mathrm{s}\right)$ and InAs $\left(\mu_{\text {Hall }} \sim 22000 \mathrm{~cm}^{2} / \mathrm{V} \cdot \mathrm{s}\right)$ [15] at a doping concentration of $5 \times 10^{16}-3 \times 10^{17} \mathrm{~cm}^{-3}$, which is around the electron density in our unintentionally doped samples. Hence, it is promising to further enhance the mobility by increasing the $\mathrm{Sb}$ content of the channel, although this comes at the cost of a lower bandgap.

Next, the electrical properties of top-gated InAsSb XOI FETs are explored. As shown in Fig. 4(a) and (b), a 7-nm-thick InAsSb FET $\left(L_{G}=500 \mathrm{~nm}\right)$ exhibits $I_{\mathrm{ON}} / I_{\mathrm{OFF}} \sim 2 \times 10^{2}$ when defining $I_{\mathrm{OFF}}$ at $V_{T}-1 / 3 V_{\mathrm{DD}}$ and $I_{\mathrm{ON}}$ at $V_{T}+2 / 3 V_{\mathrm{DD}}$ at room temperature $\left(V_{T}\right.$ is taken at $\left.I=10^{-6} \mathrm{~A} / \mu \mathrm{m}\right)$ and exhibits an $I_{\mathrm{ON}}$ of $\sim 0.38 \mathrm{~mA} / \mu \mathrm{m}$ at $V_{\mathrm{DS}}=V_{\mathrm{GS}}=0.6 \mathrm{~V}$. A subthreshold swing of $S S \sim 178 \mathrm{mV} / \mathrm{dec}$ is obtained, which is larger than that of InAs FETs $(S S \sim 125 \mathrm{mV} / \mathrm{dec})$ [16]. This suggests that the InAsSb interfaces exhibit a higher density of trap states than InAs. The source contact resistance $R_{S}$ of the 7-nm-thick InAsSb was extracted using the transmission line method. The extracted $R_{S}$ is $\sim 200 \Omega \cdot \mu \mathrm{m}$, which is close to that of the 8-nm InAs FETs $(\sim 230 \Omega \cdot \mu \mathrm{m})$ [16].

The extrinsic transconductance $g_{m}$ of the top-gated FET $\left(L_{G} \sim 500 \mathrm{~nm}\right)$ at $V_{\mathrm{DS}}=0.5 \mathrm{~V}$ peaked at $\sim 0.51 \mathrm{mS} / \mu \mathrm{m}$. The intrinsic transconductance

$$
g_{\mathrm{mi}}=g_{m} /\left(1-g_{m} R_{S}-g_{d} R_{\mathrm{SD}}\right)
$$

was extracted to exclude the contact resistance effects $\left(R_{\mathrm{SD}}=\right.$ $\left.R_{S}+R_{D}=2 R_{S}\right)$. The peak intrinsic transconductance of the device is $\sim 0.56 \mathrm{mS} / \mu \mathrm{m}$. Fig. 4(c) shows $g_{m}$ and $g_{\mathrm{mi}}$ as a 

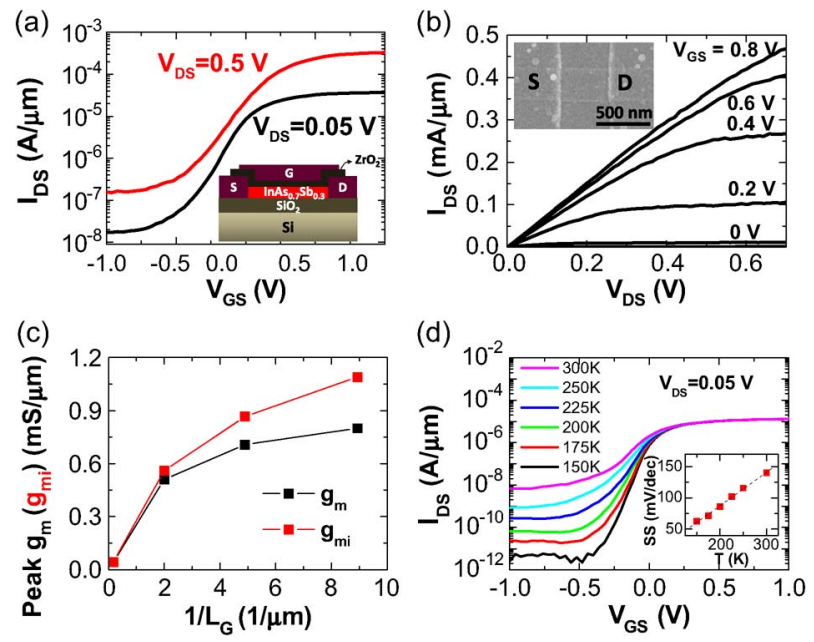

Fig. 4. (a) Top-gate $I_{\mathrm{DS}}-V_{\mathrm{GS}}$ for a 7-nm-thick $\mathrm{InAs}_{0.7} \mathrm{Sb}_{0.3} \mathrm{XOI} \mathrm{n}$-FET at $V_{\mathrm{DS}}=0.05$ and $0.5 \mathrm{~V}$. The gate length is $\sim 500 \mathrm{~nm}$. Inset shows schematic of top-gated InAsSb XOI FETs. (b) $I_{\mathrm{DS}}-V_{\mathrm{DS}}$ curve of the same device in (a). Inset shows the top-view SEM image (false color) of a representative device. (c) $g_{m}$ and $g_{\mathrm{mi}}$ as a function of the gate length. (d) Top-gate $I_{\mathrm{DS}}-V_{\mathrm{GS}}$ as a function of temperature at $V_{\mathrm{DS}}=0.05 \mathrm{~V}$. Inset shows $S S$ as a function of $T$, with linear fitting.

function of inverse gate length, which exhibits nonlinearity for shorter channel lengths, possibly arising from quasi-ballistic transport. Further study needs to be done to improve the $I_{\mathrm{OFF}}$ and $S S$ of sub-500-nm-channel-length devices. Compared to previously reported $\operatorname{InAs}_{0.8} \mathrm{Sb}_{0.2}$ QWFETs $\left(L_{G}=1 \mu \mathrm{m}\right.$ and $\left.g_{\mathrm{mi}}=0.50 \mathrm{mS} / \mu \mathrm{m}\right)$ on GaAs substrates and InSb QWFETs $\left(L_{G}=85 \mathrm{~nm}\right.$ and $\left.g_{\mathrm{mi}}=0.71 \mathrm{mS} / \mu \mathrm{m}\right)$ on Si, our InAsSb XOI FETs show a peak $g_{\mathrm{mi}}$ of $\sim 0.56 \mathrm{mS} / \mu \mathrm{m}$ for $L_{G} \sim 500 \mathrm{~nm}$ [Fig. 4(c)] while eliminating the complexity from growing thick buffer and $\delta$ doping layers [9], [10]. Moreover, it has higher $I_{\mathrm{ON}} / I_{\mathrm{OFF}}$ at room temperature.

Fig. 4(d) shows the temperature-dependent transfer characteristics. The interface trap density $\left(D_{\mathrm{it}}\right)$ was extracted from $D_{\text {it }}=C_{\text {it }} / q^{2}$, with $C_{\text {it }}$ from

$$
\frac{d S S}{d T}=\frac{2.3 k}{q}\left(1+\frac{C_{\mathrm{it}}}{C_{\mathrm{ox} 1}}+\frac{C_{\mathrm{InAsSb}}}{C_{\mathrm{ox} 1}}-\frac{\frac{C_{\mathrm{InAsSb}}^{2}}{C_{\mathrm{ox} 1} C_{\mathrm{ox} 2}}}{1+\frac{C_{\mathrm{it}}}{C_{\mathrm{ox} 2}}+\frac{C_{\mathrm{InAsSb}}}{C_{\mathrm{ox} 2}}}\right) .
$$

With $\varepsilon_{\mathrm{ox} 1}=16, t_{\mathrm{ox} 1}=10 \mathrm{~nm}, \varepsilon_{\mathrm{ox} 2}=3.9, t_{\mathrm{ox} 2}=1200 \mathrm{~nm}$, $\varepsilon_{\text {InAsSb }}=15.7$, and $t_{\text {InAsSb }}=7 \mathrm{~nm}, D_{\text {it }}$ is determined to be $\sim 1 \times 10^{13} \mathrm{~cm}^{-2} \mathrm{eV}^{-1}$, which is slightly higher than that of InAs XOI FETs $\left(\sim 3 \times 10^{12} \mathrm{~cm}^{-2} \mathrm{eV}^{-1}\right)$ [16]. Note that this $D_{\text {it }}$ value presents only a rough estimate of the order of magnitude for the average trap density within the bandgap. The traps close to or beyond the conduction band edge are not extracted using this analysis technique, but they can also alter the charge carrier density and transport properties. Detailed capacitance-voltage characterization in the future is needed to better understand and optimize the surface/interface properties.

\section{CONCLUSiON}

In conclusion, high-electron-mobility $\mathrm{InAs}_{0.7} \mathrm{Sb}_{0.3}$ transistors have been fabricated on Si substrates using the XOI configuration. The devices exhibit excellent electrical properties, while future work on improving the InAsSb/high- $\kappa$ interface needs to be done. In the future, even higher $\mathrm{Sb}$ content and thinner body InAsSb XOI n-FETs, together with InGaSb XOI p-FETs, are promising to be integrated for high-speed and lowpower complementary MOSFET circuits.

\section{ACKNOWLEDGMENT}

H. Fang and S. Chuang contributed equally to this work.

\section{REFERENCES}

[1] R. Chau, S. Datta, M. Doczy, B. Doyle, B. Jin, J. Kavalieros, A. Majumdar, M. Metz, and M. Radosavljevic, "Benchmarking nanotechnology for high-performance and low-power logic transistor applications," IEEE Trans. Nanotechnol., vol. 4, no. 2, pp. 153-158, Mar. 2005.

[2] D. A. Antoniadis and A. Khakifirooz, "MOSFET performance scaling: Limitations and future options," in IEEE IEDM Tech. Dig., 2008, pp. 1-4.

[3] D.-H. Kim and J. A. del Alamo, "Scalability of sub-100 nm InAs HEMTs on InP substrate for future logic applications," IEEE Trans. Electron Devices, vol. 57, no. 7, pp. 1504-1511, Jul. 2010.

[4] Y. Xuan, Y. Q. Wu, T. Shen, T. Yang, and P. D. Ye, "High performance submicron inversion-type enhancement-mode InGaAs MOSFETs with ALD $\mathrm{Al}_{2} \mathrm{O}_{3}, \mathrm{HfO}_{2}$ and $\mathrm{HfAlO}$ as gate dielectrics," in IEEE IEDM Tech. Dig., 2007, pp. 637-640.

[5] B. R. Bennett, R. Magno, J. B. Boos, W. Kruppa, and M. G. Ancona, "Antimonide-based compound semiconductors for electronic devices: A review," Solid State Electron., vol. 49, no. 12, pp. 1875-1895, Dec. 2005.

[6] A. Ali, H. Madan, R. Misra, A. Agrawal, P. Schiffer, J. B. Boos, B. R. Bennett, and S. Datta, "Experimental determination of quantum and centroid capacitance in arsenide-antimonide quantum-well MOSFETs incorporating nonparabolicity effect," IEEE Trans. Electron Devices, vol. 58, no. 5, pp. 1397-1403, May 2011.

[7] B.-R. Wu, C. Liao, and K. Y. Cheng, "High quality InAsSb grown on InP substrates using AlSb/AlAsSb buffer layers," Appl. Phys. Lett., vol. 92, no. 6, pp. 062111-1-062111-3, Feb. 2008.

[8] S. Datta, T. Ashley, J. Brask, L. Buckle, M. Doczy, M. Emeny, D. Hayes, K. Hilton, R. Jefferies, T. Martin, T. J. Phillips, D. Wallis, P. Wilding, and R. Chau, "85 nm gate length enhancement and depletion mode InSb quantum well transistors for ultra high speed and very low power digital logic applications," in IEEE IEDM Tech. Dig., 2005, pp. 763-766.

[9] A. Ali, H. Madan, R. Misra, E. Hwang, A. Agrawal, I. Ramirez, P. Schiffer, T. N. Jackson, S. E. Mohney, J. B. Boos, B. R. Bennett, I. Geppert, M. Eizenberg, and S. Datta, "Advanced composite high- $\kappa$ gate stack for mixed anion arsenide-antimonide quantum well transistors," in IEEE IEDM Tech. Dig., 2010, pp. 6.3.1-6.3.4.

[10] T. Ashley, L. Buckle, S. Datta, M. T. Emeny, D. G. Hayes, K. P. Hilton, R. Jefferies, T. Martin, T. J. Phillips, D. J. Wallis, P. J. Wilding, and R. Chau, "Heterogeneous InSb quantum well transistors on silicon for ultra-high speed, low power logic applications," Electron. Lett., vol. 43, no. 14, Jul. 2007.

[11] H. Ko, K. Takei, R. Kapadia, S. Chuang, H. Fang, P. W. Leu, K. Ganapathi, E. Plis, H. S. Kim, S.-Y. Chen, M. Madsen, A. C. Ford, Y.-L. Chueh, S. Krishna, S. Salahuddin, and A. Javey, "Ultrathin compound semiconductor on insulator layers for high-performance nanoscale transistors," Nature, vol. 468, no. 7321, pp. 286-289, Nov. 2010.

[12] Y.-K. Choi, K. Asano, N. Lindert, V. Subramanian, T.-J. King, J. Bokor, and C. Hu, "Ultra-thin body SOI MOSFET for deep-sub-tenth micron era," in IEEE IEDM Tech. Dig., 1999, pp. 919-921.

[13] H. T. Grahn, Introduction to Semiconductor Physics. Singapore: World Scientific, 1999, p. 121.

[14] K. Takei, H. Fang, S. B. Kumar, R. Kapadia, Q. Gao, M. Madsen, H. S. Kim, C.-H. Liu, Y.-L. Chueh, E. Plis, S. Krishna, H. A. Bechtel, J. Guo, and A. Javey, "Quantum confinement effects in nanoscalethickness InAs membranes," Nano Lett., vol. 11, no. 11, pp. 5008-5012, 2011.

[15] D. Chattopadhyay, S. K. Sutradhar, and B. R. Nag, "Electron transport in direct-gap III-V ternary alloys," J. Phys. C, Solid State Phys., vol. 14 no. 6, pp. 891-908, Feb. 1981.

[16] K. Takei, S. Chuang, H. Fang, R. Kapadia, C.-H. Liu, J. Nah, H. S. Kim, E. Plis, S. Krishna, Y.-L. Chueh, and A. Javey, "Benchmarking the performance of ultrathin body InAs-on-insulator transistors as a function of body thickness," Appl. Phys. Lett., vol. 99, no. 10, pp. 103507-1103507-3, Sep. 2011. 